\title{
Clinicopathological Behavior and Oncological Outcomes of Malignant Parotid Tumors in a Pakistani Population
}

Muhammad Faisal ${ }^{1}$, Taskheer Abbas ${ }^{2}$, Mohammad Adeel ${ }^{3}$, Usman Khaleeq ${ }^{2}$, Abdul Wahid Anwer $^{4}$, Kashif Malik ${ }^{1}$, Raza Hussain ${ }^{5}$, Arif Jamshed ${ }^{6}$

1. Department of Surgical Oncology, Shaukat Khanum Memorial Cancer Hospital and Research Center, Lahore, PAK 2. Radiation Oncology, Shaukat Khanum Memorial Cancer Hospital and Research Center, Lahore, PAK 3. Department of Surgical Oncology, Shaukat Khanum Memorial Cancer Hospital and Research Center, Lahore, Pakistan 4. Department of Surgical Oncology, Shaukat Khanum Memorial Cancer Hospital and Research Center, Lahore, Pakistan, Lahore, PAK 5. Department of Surgical Oncology, Shaukat Khanum Memorial Cancer Hospital and Research Center, Lahore, Pakistan, lahore, PAK 6. Department of Radiation Oncology, Shaukat Khanum Memorial Cancer Hospital and Research Center, Lahore, PAK

$\square$ Corresponding author: Muhammad Faisal, muhammadfaisal5767@outlook.com Disclosures can be found in Additional Information at the end of the article

\section{Abstract}

\section{Introduction}

The incidence of salivary gland tumors is influenced by geographical and racial factors resulting in diverse histology. While salivary gland tumors account for a low proportion of head and neck cancers, most malignant tumors of the salivary gland are located in the parotid gland. The goals of this study are to describe the clinicopathological behavior of malignant parotid tumors and explore oncological outcomes related to survival in our Pakistani tertiary care cancer hospital.

\section{Methods}

We conducted a retrospective analysis of 209 patients diagnosed with malignant parotid tumors from 2004 to 2016. Data such as demographics, age, gender, histology, grade, clinical and pathological stage, surgical treatment types and adjuvant modalities used were analyzed using SPSS software version 20. We used Kaplan Meier curves to analyze survival data.

\section{Results}

Received 11/01/2017

Review began 01/28/2018 Review ended 02/01/2018 Published 02/05/2018

\section{(c) Copyright 2018}

Faisal et al. This is an open access article distributed under the terms of the Creative Commons Attribution License CC-BY 3.0., which permits unrestricted use, distribution, and reproduction in any medium, provided the original author and source are credited.
The median patient age at diagnosis was 40 years, and the ratio of men to women was 1.2:1. Mucoepidermoid carcinoma was the most common histological variant (with a $50 \%$ incidence rate) followed by adenoid cystic carcinoma (13\%), and adenocarcinoma (10\%). Histology has further categorized these malignant tumors into low (34\%), intermediate (28\%), and high (21\% ) grades. The American Joint Committee on Cancer, seventh edition, clinical staging was Stage I (21\%), II (28\%), III (15\%), and IV (34\%). The 5-year survival was 68\%, and the 10 -year survival was $45 \%$.

\section{Conclusion}

Mucoepidermoid carcinoma is the most common malignant parotid histology in our patient population. Advanced age, increased T stage (size $>4 \mathrm{~cm}$ ), high-grade histology, and cervical nodal involvement decrease overall survival. Open biopsies, piecemeal excisions, and delayed 
presentation for radiotherapy post-surgery may also have role in adverse outcomes in these malignancies.

Categories: Otolaryngology, Oncology, Other

Keywords: salivary gland neoplasms, malignant parotid tumors, survival outcome

\section{Introduction}

Malignant tumors of the parotid gland are characterized by their low incidence rate (1\% to 3\% of all head and neck cancers) and marked histopathological heterogeneity [1]. Approximately $70 \%$ of the malignant tumors of the major salivary glands are in the parotid gland [2]. Tumors in the submandibular and parotid regions are not quite as accessible. For these tumors, fineneedle aspiration (FNA) is the preferred method of biopsy. Several studies have now demonstrated that FNA is a reliable technique achieving a diagnostic result in $88 \%$ to $98 \%$ of biopsies [3-5]. Superficial parotidectomy preserving the facial nerve has remained the recommended treatment modality, while adjuvant treatment and cervical lymph node removal are usually reserved for advanced stage, high-grade histology tumors [6-8]. Factors influencing the oncological outcome of these tumors include the level of histological diversity, primary tumor status, regional nodal involvement, and adjuvant use of chemo-radiotherapy. Our study aims to describe the clinicopathological behavior of malignant parotid tumors and explore the oncological outcomes related to survival from a tertiary care cancer hospital in Pakistan.

\section{Materials And Methods}

We conducted a retrospective analysis of 209 patients with a malignant parotid tumor who received treatment from January 2004 to December 2014 at Shaukat Khanum Memorial Cancer Hospital and Research Center in Lahore, Pakistan.

All patients presented to the head and neck clinic after histological confirmation by FNA cytology. Imaging such as computerized tomography or magnetic resonance imaging was utilized to assess the local extent of the disease as well as the nodal status.

A head and neck multi-disciplinary team discussed each patient before making a final decision on treatment.

Surgical procedures performed were superficial parotidectomy (nerve sparing), completion parotidectomy (operation following previous incomplete procedure), radical parotidectomy (nerve sacrificing), extended radical parotidectomy (sacrificing adjacent muscles and soft tissue such as skin depending upon local extension of the disease). Neck dissection as part of the procedure was offered to those with tumor size of $>3 \mathrm{~cm}$, clinically involved lymph nodes, nodes found to be positive during intraoperative frozen sections, and high-grade malignancy.

Similarly, adjuvant radiotherapy was offered to those with high-grade malignancy, close or positive resection margins, and tumor size $>4 \mathrm{~cm}$, and positive lymph nodes.

Variables such as patient age, gender, clinical and pathological staging, final histology, grading, type of treatment modality used, the extent of neck dissection, margin status, and site of recurrence were recorded and evaluated. We have grouped patients into early (T1-T2) and locally advanced (T3-T4) disease stages to avoid dispersing the data. Patients were also categorized into clinically node negative and positive as well as pathologically node negative and positive groups to analyze survival. 


\section{Cureus}

Data were analyzed using IBM SPSS Statistics for Windows, Version 20.0 (released 2011, IBM Corp. Armonk, NY). Kaplan Meier curves were used to analyze survival data.

The study was reviewed by the Institutional Review Board, and the exemption was granted by the Ethical Review Committee of Shaukat Khanum Memorial Cancer Hospital and Research Center, Lahore, Pakistan.

\section{Results}

We retrospectively analyzed the medical records of 209 patients diagnosed with malignant parotid neoplasms. There were 119 men and 94 women. One hundred sixty-four patients were primarily treated with surgery or surgery followed by radiotherapy in our hospital while 39 patients underwent operations in a different facility but were given adjuvant treatment in our clinic. The median patient age at diagnosis was 40 years. The median follow-up was 24 months. Table 1 shows the clinical and pathological staging, histology, type of parotidectomy, and treatment modality used. Clinical tumor stage showed cT1 (49 cases), cT2 (65 cases), cT3 (31 cases), and cT4 (64 cases). Pathological classification showed pT1 (43 cases), pT2 (60 cases), pT3 (38 cases), and pT4 (23 cases).

Eighty-two patients received superficial parotidectomy, 71 patients received complete parotidectomy, nine patients received radical parotidectomy, and two patients received extended radical parotidectomy.

Forty-one patients received neck dissection as part of the procedure, and 36 of those were classified as $\mathrm{pN}+$ while five were classified $\mathrm{pN} 0$.

Histology revealed mucoepidermoid carcinoma to be the most common malignancy (with a 50\% incidence rate) followed by adenoid cystic carcinoma (10.5\%) and adenocarcinoma (9.6\%). Only 23 patients received surgery alone while 141 patients received surgery followed by radiotherapy. Thirty-nine patients underwent operations in a different facility, and these patients received postoperative radiotherapy in the presence of adverse conditions such as a close/positive margin, high-grade malignancy, tumor size $>4 \mathrm{~cm}$, facial nerve involvement, and extraparenchymal extension. The median postoperative radiotherapy dose was $60 \mathrm{~Gy}$ on the parotid gland and 55 Gy on the neck. Adjuvant chemotherapy was used in select patients who had gross lymph nodal involvement.

The five-year and 10-year overall survival was $68 \%$ and $45 \%$, respectively, for all the patients (Figure 1). The five-year and 10-year survival rates were $78 \%$ and $54 \%$, respectively, for those treated exclusively in our hospital (Figure 2). Variables, which have statistical significance on survival, were age (categorized as either $<50$ or $>50$ years; $\mathrm{p}=0.029$ ), grade (high vs low; $\mathrm{p}<$ .001 ), pathological nodal involvement ( $\mathrm{pN} 0 \mathrm{vs} \mathrm{pN}+; \mathrm{p}<.001$ ), and pathological tumor size (pT1-2 vs pT3-4; $<$ < 0.001) as shown in Table 2 and Figures 3-5. Multivariate analysis showed age above 50 years, high grading, advanced pathological stage, and nodal involvement to be significant factors affecting survival (Table 3). The 5-year survival of patients who were previously treated outside our hospital has not shown promising results in terms of survival (Figure 6).

Age

$<50$ years
$\mathbf{N}$

$\%$

141

67.5 


\section{Cureus}

Sex

Male

cT

pT

Surgical treatment type

Superficial parotidectomy

Extended radical parotidectomy

\section{Histology}

Mucoepidermoid carcinoma 


\section{Cureus}

Treatment modality

Surgery

Surgery + Adjuvant radiotherapy

Adjuvant radiotherapy

Neck dissection

Yes

No

TABLE 1: Clinico-pathological characteristics.

\begin{tabular}{|c|c|c|c|}
\hline & 5-Year Survival & 10-Year Survival & $p$-value \\
\hline Age $<50$ years & $82 \%$ & $58 \%$ & \multirow{2}{*}{.029} \\
\hline$>50$ years & $62 \%$ & $51 \%$ & \\
\hline Male & $77 \%$ & $51 \%$ & \multirow{2}{*}{0.57} \\
\hline Female & $77 \%$ & $61 \%$ & \\
\hline с T1-T2 & $86 \%$ & $79 \%$ & \multirow{2}{*}{$<0.0001$} \\
\hline c T3-T4 & $43 \%$ & $14 \%$ & \\
\hline p T1-T2 & $88 \%$ & $71 \%$ & \multirow{2}{*}{$<0.0001$} \\
\hline p T3-T4 & $58 \%$ & $25 \%$ & \\
\hline c N0 & $77 \%$ & $65 \%$ & \multirow{2}{*}{$<0.0001$} \\
\hline $\mathrm{cN}+$ & $33 \%$ & $0 \%$ & \\
\hline p NO & $85 \%$ & $73 \%$ & \multirow{2}{*}{$<0.0001$} \\
\hline $\mathrm{p} \mathrm{N+}$ & $55 \%$ & $0 \%$ & \\
\hline ND: Yes & $74 \%$ & $74 \%$ & \multirow{2}{*}{$<0.0001$} \\
\hline ND: No & $85 \%$ & $79 \%$ & \\
\hline \multicolumn{4}{|l|}{ Grade } \\
\hline High & 56 & 30 & \multirow{3}{*}{$<0.001$} \\
\hline Low & 87 & 70 & \\
\hline PNI & & & \\
\hline
\end{tabular}




\section{Cureus}

Yes

No

Extra-parenchymal extension

Yes

No

Adjuvant PORT

Yes

No
$56 \%$

$77 \%$

$66 \%$

$75 \%$

$76 \%$

$90 \%$

$0 \%$

$58 \%$

$0 \%$

0.150

$54 \%$

$49 \%$

\section{TABLE 2: Prognostic factors affecting survival outcome.}

ND: Neck dissection; cN0: Clinically negative neck nodes; cN+: Clinically positive/palpable nodes neck; pN0: Pathologically negative neck nodes; pN+: Pathologically positive neck nodes; PNI: Perineural invasion; PORT: Post operative radiotherapy.

\begin{tabular}{|c|c|c|}
\hline Variable & p-value & Hazard Ratio \\
\hline Age ( $<50$ years vs $>50$ years) & 0.127 & 1.8 \\
\hline Grade (Low vs High) & 0.05 & 2.1 \\
\hline pT1-2 vs pT3-4 & 0.02 & 2.6 \\
\hline pN0 vs pN+ & 0.01 & 2.7 \\
\hline
\end{tabular}

TABLE 3: Multivariate analysis affecting survival outcome. 


\section{Cureus}

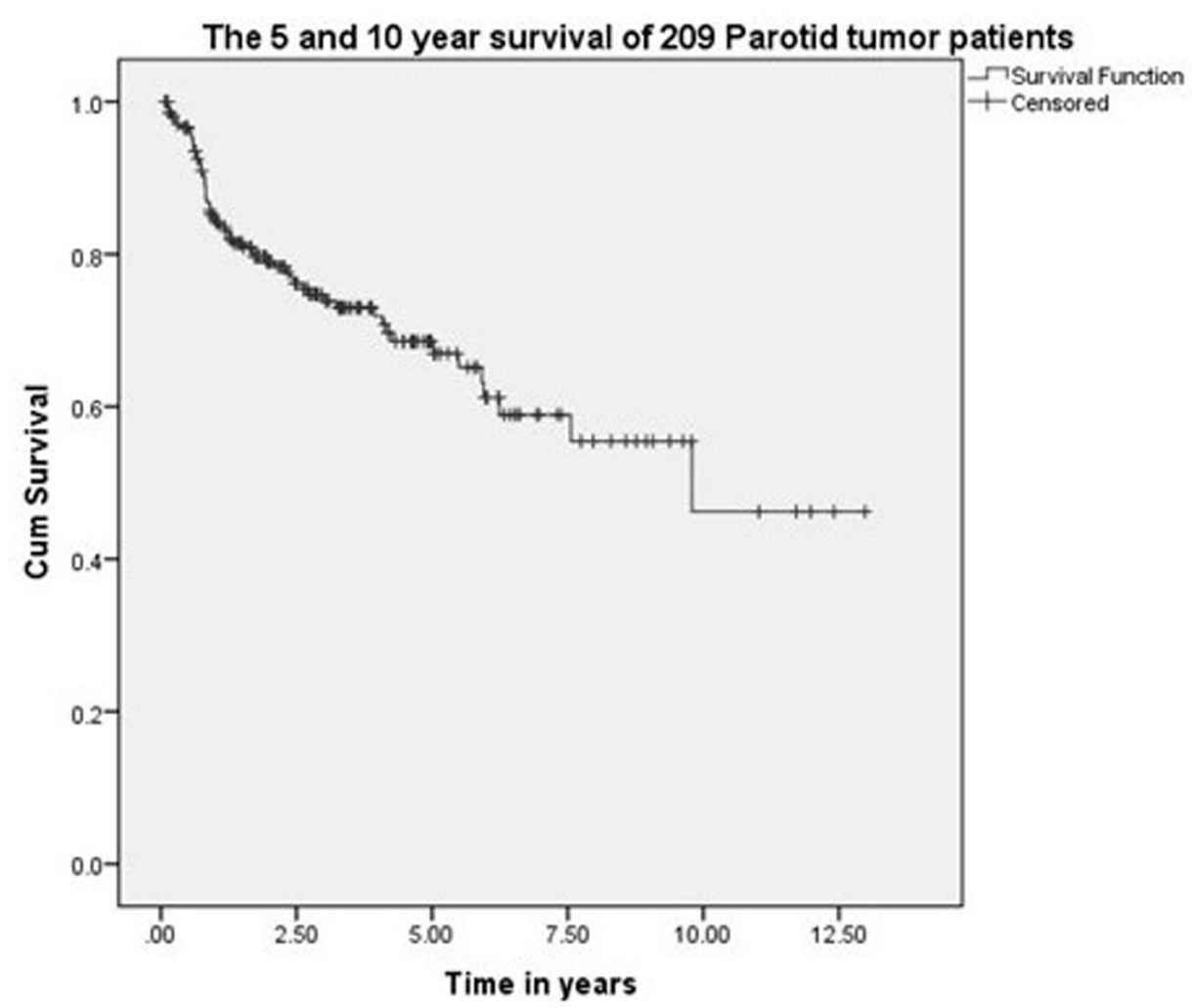

FIGURE 1: 5- and 10-year survival of 209 malignant parotid tumor patients. 


\section{Cureus}

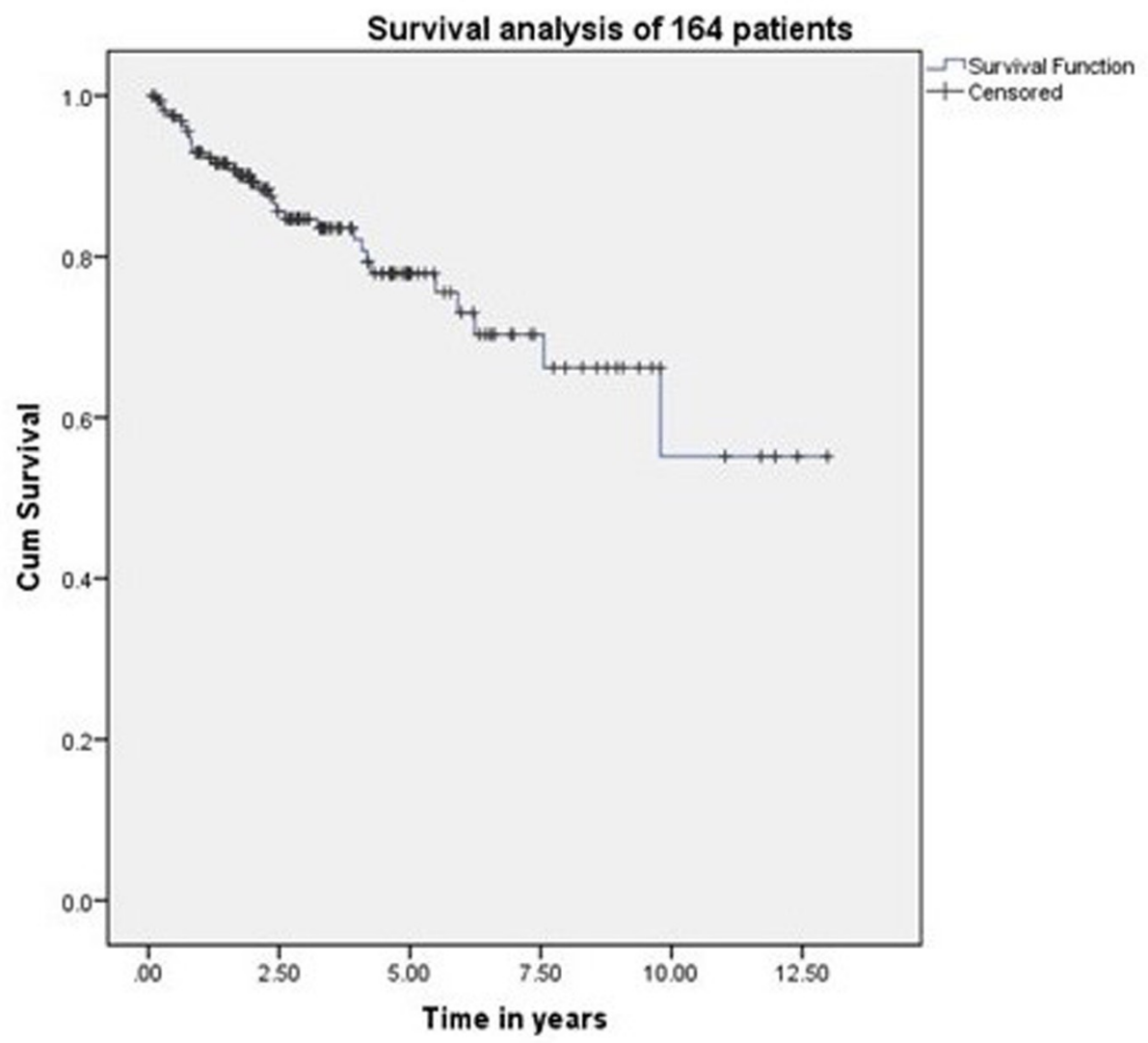

FIGURE 2: Survival of 164 patients treated with surgery and radiotherapy. 


\section{Cureus}

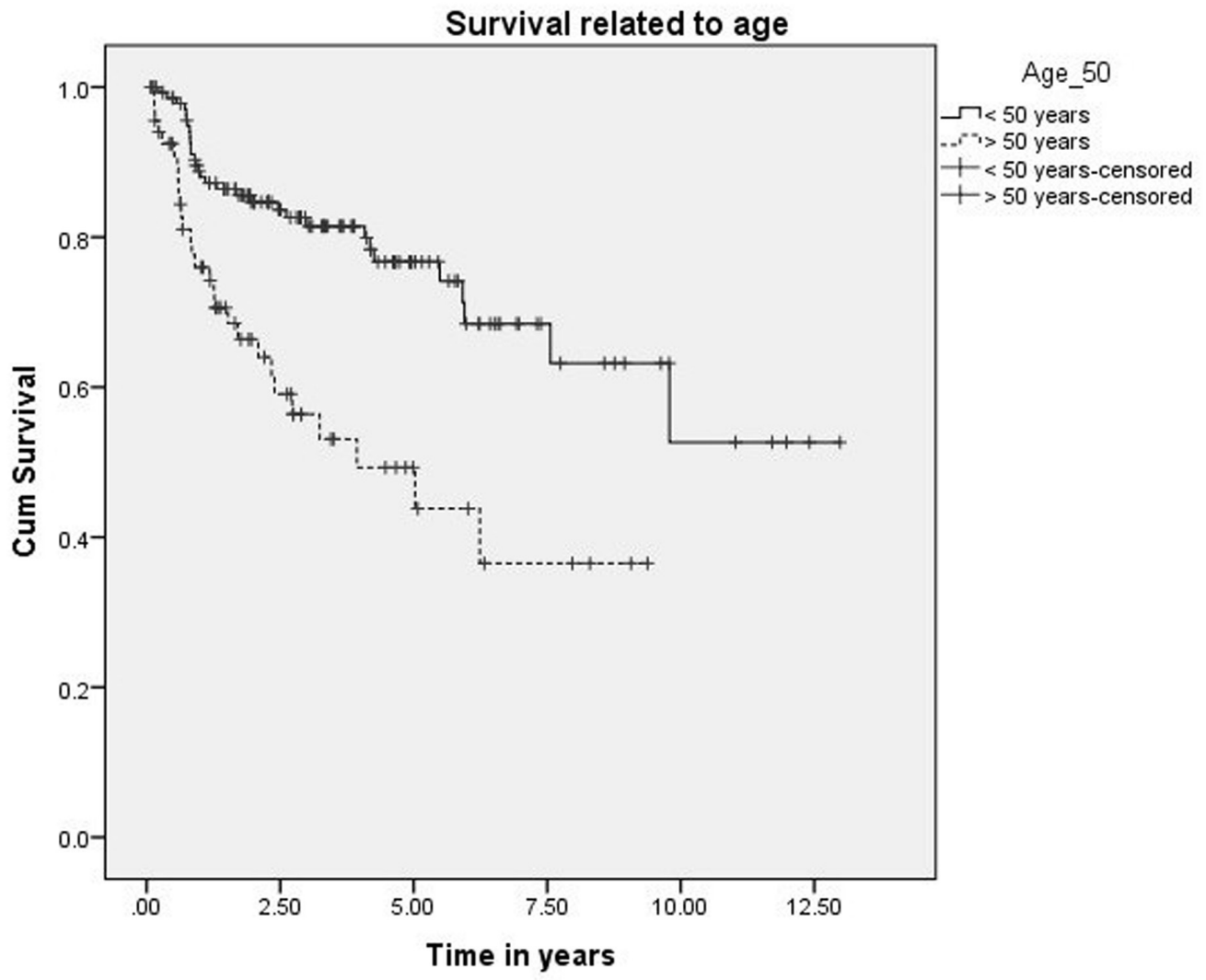

FIGURE 3: Survival related to age. 


\section{Cureus}

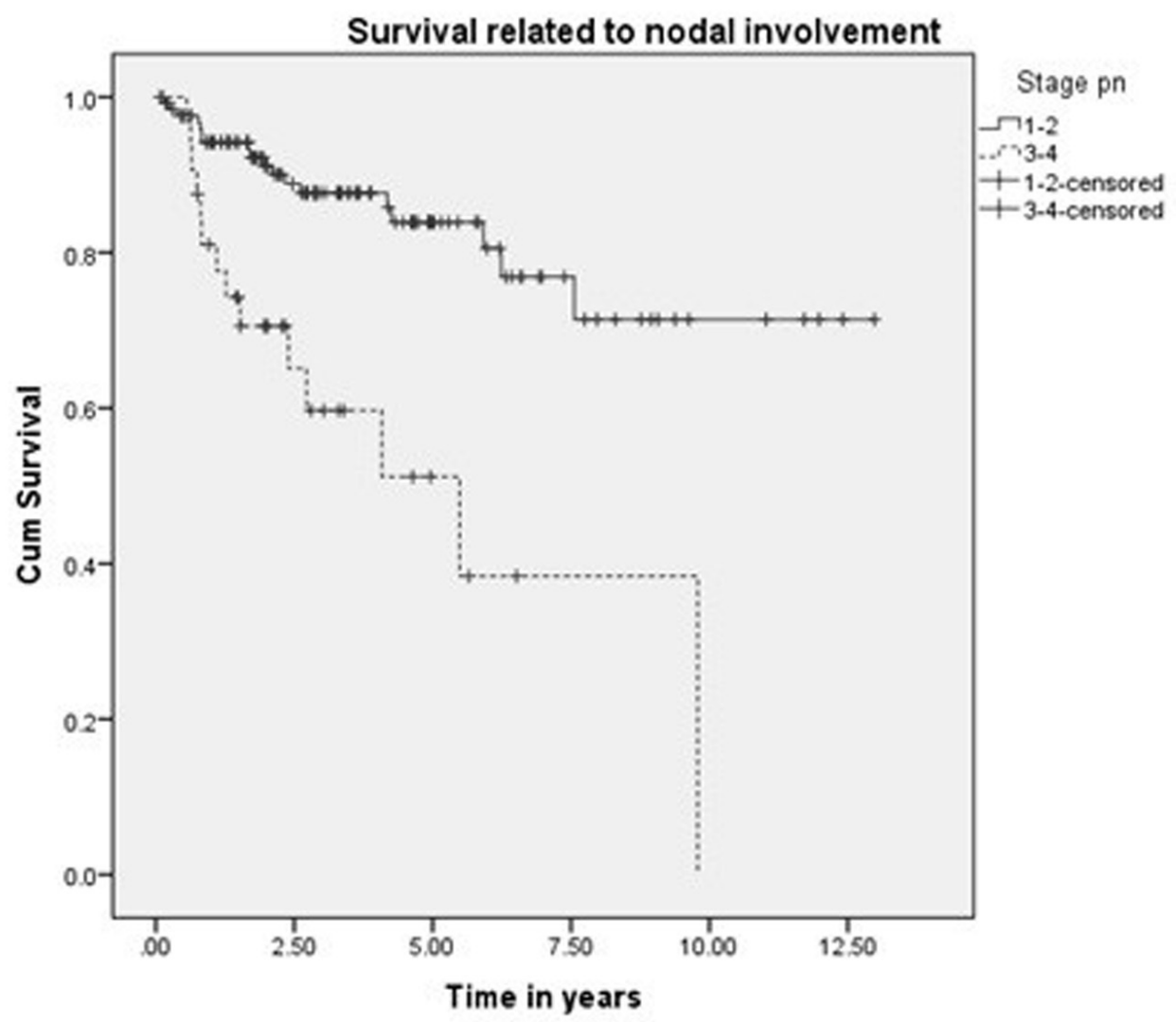

FIGURE 4: Survival related to nodal stage. 


\section{Cureus}

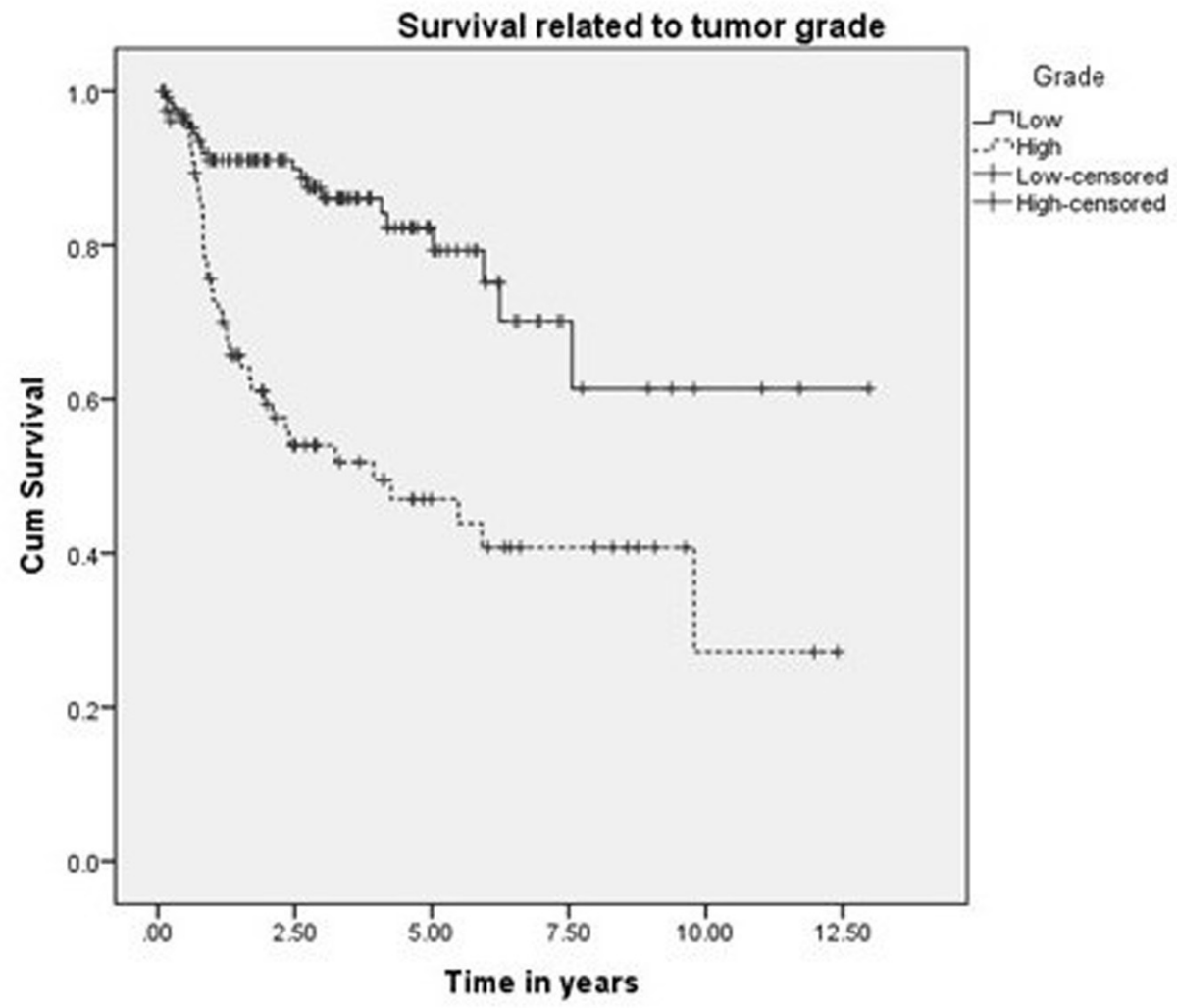

FIGURE 5: Survival related to grade. 


\section{Cureus}

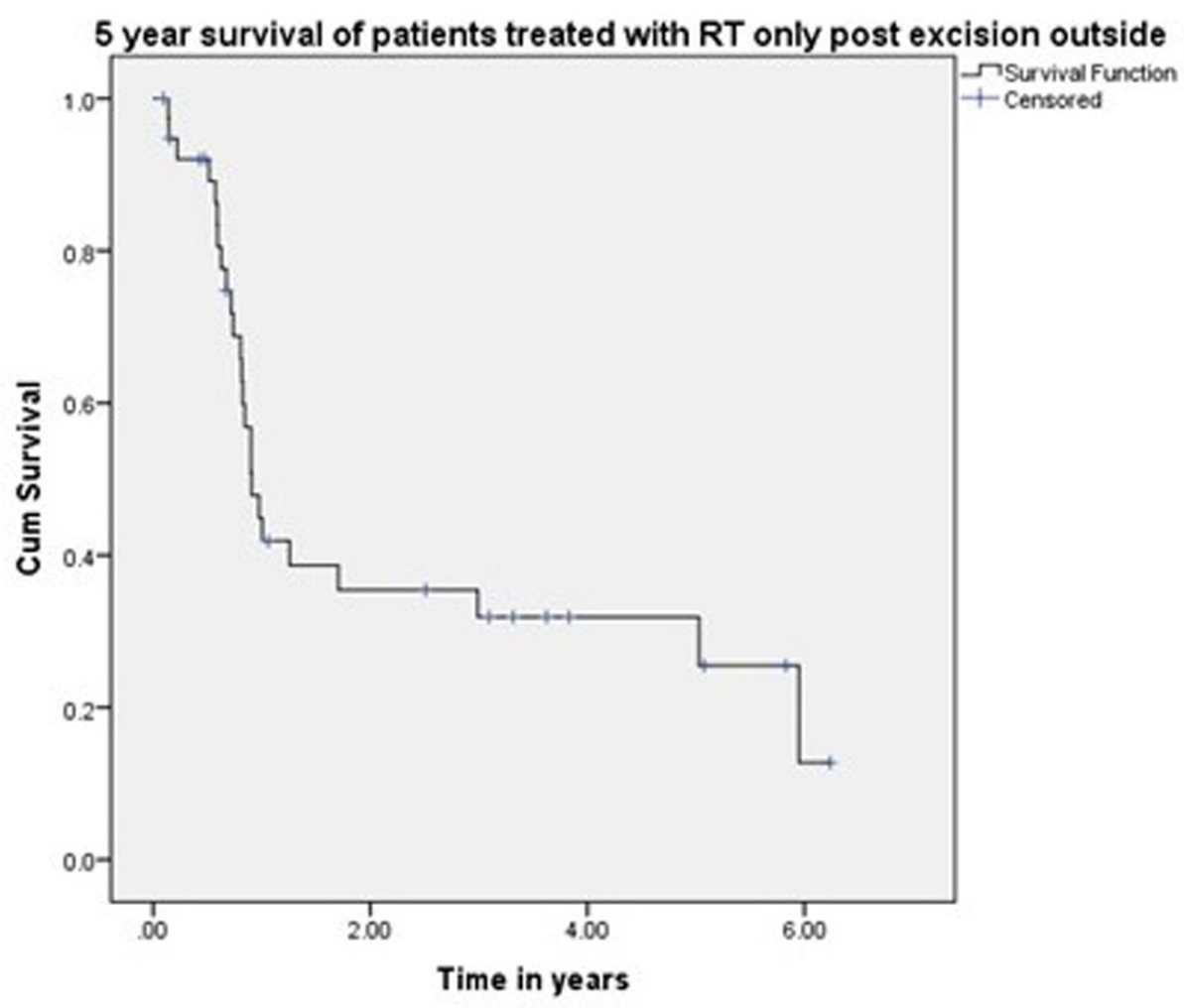

FIGURE 6: Survival of patients treated outside.

\section{Discussion}

Most salivary neoplasms originate in the parotid gland, while minor salivary gland and submandibular gland neoplasms are much less common. A low incidence combined with histological heterogeneity poses a challenge in evaluating survival and oncological outcomes [9].

Age and gender have been postulated as prognostic factors in studies published by Malata, et al., Pohar, et al., Koul, et al., Jouzdani, et al., and Lima, et al., in contrary to a study by Mercante, et al. reporting no difference in survival [10-15]. Based on our findings, age is an important prognostic factor in terms of survival $(\mathrm{p}=0.029)$, but no significant difference was found as far as gender is concerned (Figure 3).

Harbo, et al. established a relationship related to tumor stage and histological classification [16]. A retrospective study on 152 patients with parotid tumors found a statistically significant difference in survival based on tumor stage. The survival rate for Stage I and II tumors was 65\% and 50\%, respectively, and falls to $9 \%$ in Stage IV patients [17]. The prognosis for survival in histologically well-differentiated tumors (52\%) was also better than the prognosis associated with poorly differentiated lesions (19\%). Our experience showed that survival related to malignant parotid tumors depends upon cT ( $p<0.0001), \mathrm{pT}(\mathrm{p}<0.0001), \mathrm{cN}(\mathrm{p}<0.0001), \mathrm{pN}$ ( $\mathrm{p}$ $<0.0001)$, histology ( $<<0.001)$, and ND ( $<<0.0001)$, which is comparable to other retrospective series as demonstrated by Lima, et al., Terhaard, et al., and Stenner, et al. [14,17,18]. Thus, patients with clinical and pathological tumor sizes $>$ T2, high-grade histology, clinical and 
pathological nodal involvement, and perineural involvement have worse five-year and 10-year survival (Figure 4 and Figure 5). The role of adjuvant radiotherapy is reported to improve survival according to Armstrong, et al. and Mendenhall, et al. [19-20], but our results indicated no such benefit (Figure 2). Postoperative radiation therapy may reduce the risk of loco-regional failure, thus decreasing the risk of damage to the facial nerve in cases of loco-regional failures. Clear evidence of a dose-response is lacking in this rare disease, and the dose of 60 Gy in daily 2 Gy fractions represents a practical consensus. Developments in radiotherapy technique such as intensity modulated radiotherapy have been applied to parotid treatment [21,22]. A careful analysis of our data has shown that patients with adverse features were candidates for adjuvant radiotherapy, which may explain the poor survival outcome in these individuals. As the only tertiary care referral cancer hospital, we cannot refuse patients who have been previously treated elsewhere with inadequate resections. Of 209 malignant parotid tumor patients, 39 had been accepted for adjuvant treatment after surgery in a facility outside of our hospital. The five-year survival of these individuals was only 25\% (Figure ๑). In our experience, this poor outcome is mostly attributed to open biopsies outside contaminating the surrounding bed by spillage of tumor cells, fragmented nature of the resected specimen, delayed presentations for adjuvant treatment post-primary surgery and attempting inoperable tumors leaving behind gross residual disease by outside surgeons. Similarly, the role of extraparenchymal extension affecting survival has been described by Klussmann, et al., but its role is not statistically evident according to our results. This may be due to only a small number of patients having extraparenchymal extension, and larger sample size may highlight its role in survival [23].

\section{Conclusions}

We evaluated oncological outcomes of malignant parotid salivary gland tumors by identifying the prognostic factors affecting survival. Advanced age, high-grade histology, increased size (>T2) and clinicopathological nodal involvement adversely affect overall survival. Adjuvant radiotherapy, however, has not resulted in improved survival particularly in the background of advanced disease.

\section{Additional Information \\ Disclosures}

Human subjects: Consent was obtained by all participants in this study. Shaukat Khanum Memorial Cancer Hospital and Research Center Review Board issued approval N/A. The study has been reviewed by Institutional Review Board and exemption was granted from Ethical Review Committee of Shaukat Khanum Memorial Cancer Hospital and Research Center, Lahore, Pakistan. Animal subjects: All authors have confirmed that this study did not involve animal subjects or tissue. Conflicts of interest: In compliance with the ICMJE uniform disclosure form, all authors declare the following: Payment/services info: All authors have declared that no financial support was received from any organization for the submitted work. Financial relationships: All authors have declared that they have no financial relationships at present or within the previous three years with any organizations that might have an interest in the submitted work. Other relationships: All authors have declared that there are no other relationships or activities that could appear to have influenced the submitted work.

\section{Acknowledgements}

Thanks to Dr. Arif Jamshed and Dr. Raza Hussain for their support and guidance.

\section{References}

1. Tsai SC, Hsu HT: Parotid neoplasms: diagnosis, treatment, and intraparotid facial nerve anatomy. J Laryngol Otol. 2002, 116:359-362. 10.1258/0022215021911013 
2. Henau K, Frankart J, Vos K, et al.: Cancer Incidence in Belgium 2008. Van Eycken L (ed): Belgian Cancer Registry, Brussels; 2008.

3. Seethala RR, LiVolsi VA, Baloch ZW: Relative accuracy in fine needle aspiration and frozen section in the diagnosis of lesions in the parotid gland. Head Neck. 2005, 27:217-223. 10.1002/hed.20142

4. Cohen EG, Patel SG, Lin O, et al.: Fine-needle aspiration of the salivary gland lesions in a selected patient population. Arch Otolaryngol Head Neck Surg. 2004, 130:773-778. 10.1001/archotol.130.6.773

5. Postema RJ, van Velthuysen M-LF, van den Brekel MWM, et al.: Accuracy of fine-needle aspiration cytology of salivary gland lesions in the Netherlands Cancer Institute. Head Neck. 2004, 26:418-424. 10.1002/hed.10393

6. Vander Poorten VLM, Balm AJM, Hilgers FJM: Management of cancer of the parotid gland . Curr Opin Otolaryngol Head Neck Surg. 2002, 10:134-144. 10.1097/00020840-20020400000013

7. Granell J, Sánchez-Jara JL, Gavilanes J, et al.: Management of the surgical pathology of the parotid gland: a review of 54 cases (Article in Spanish). Acta Otorrinolaringol Esp. 2010, 61:189-195. 10.1016/j.otorri.2009.11.006

8. North CA, Lee D-J, Piantadosi S, et al.: Carcinoma of the major salivary glands treated by surgery or surgery postoperative radiotherapy. Int J Radiat Oncol Biol Phys. 1990, 18:13191326.

9. Spiro RH: Treating tumors of the sublingual glands, including a useful technique for repair of the floor of the mouth after resection. Am J Surg. 1995, 170:457-460. 10.1016/s00029610(99)80329-8

10. Malata CM, Camilleri IG, McLean NR, et al.: Malignant tumours of the parotid gland: a 12year review. Br J Plast Surg. 1997, 50:600-608. 10.1016/s0007-1226(97)90505-1

11. Pohar S, Gay H, Rosenbaum P, et al.: Malignant parotid tumours: presentation, clinical/pathological prognostic factors and treatment outcomes. Int J Radiat Oncol Biol Phys. 2005, 61:112-118. 10.1016/j.ijrobp.2004.04.052

12. Koul R, Dubey A, Butler J, et al.: Prognostic factors depicting disease-specific survival in parotid-gland tumors. Int J Radiat Oncol Biol Phys. 2007, 68:714-718. 10.1016/j.ijrobp.2007.01.009

13. Jouzdani E, Yachouh J, Costes V, et al.: Prognostic value of a three-grade classification in primary epithelial parotid carcinoma: result of a histological review from a 20-year experience of total parotidectomy with neck dissection in a single institution. Eur J Cancer. 2010, 46:323331. 10.1016/j.ejca.2009.10.012

14. Lima RA, Tavares MR, Dias FL, et al.: Prognostic factors in malignant parotid gland tumors . Otolaryngol Head Neck Surg. 2005, 133:702-708. 10.1016/j.otohns.2005.08.001

15. Mercante G, Marchese C, Giannarelli D, et al.: Oncological outcome and prognostic factors in malignant parotid tumours. J Craniomaxillofac Surg. 2014, 42:59-65. 10.1016/j.jcms.2013.02.003

16. Harbo G, Bundgaard T, Pedersen D, et al.: Prognostic indicators for malignant tumors of the parotid gland. Clin Otolaryngol. 2002, 27:512-516. 10.1046/j.1365-2273.2002.00625.x

17. Terhaard CHJ, Lubsen H, Rasch CRN, et al.: The role of radiotherapy in the treatment of malignant salivary gland tumors. Int J Radiat Oncol Biol Phys. 2005, 61:103-111.

10.1016/j.ijrobp.2004.03.018

18. Stenner M, Molls C, Klussmann JP, et al.: Prognosis of surgically treated primary parotid gland cancer - an evaluation of 231 cases [Article in German]. Laryngorhinootologie. 2011, 90:664671. 10.1055/s-0031-1285924

19. Armstrong JG, Harrison LB, Spiro RH, et al.: Malignant tumors of major salivary gland origin. A matched-pair analysis of the role of combined surgery and postoperative radiotherapy. Arch Otolaryngol Head Neck Surg. 1990, 116:290-293.

10.1001/archotol.1990.01870030054008

20. Mendenhall WM, Morris CG, Amdur RJ, et al.: Radiotherapy alone or combined with surgery for salivary gland carcinoma. Cancer. 2005, 103:2544-2550. 10.1002/cncr.21083

21. Nutting CM, Rowbottom CG, Cosgrove VP, et al.: Optimisation of radiotherapy for carcinoma of the parotid gland: a comparison of conventional, three-dimensional conformal, and intensity-modulated techniques. Radiother Oncol. 2001, 60:163-172. 10.1016/s01678140(01)00339-5 
Cureus

22. Bragg CM, Conway J, Robinson MH: The role of intensity-modulated radiotherapy in the treatment of parotid tumors. Int J Radiat Oncol Biol Phys. 2002, 52:729-738. 10.1016/s03603016(01)02660-8

23. Klussmann JP, Ponert T, Mueller RP, et al.: Patterns of lymph node spread and its influence on outcome in resectable parotid cancer. Eur J Surg Oncol. 2008, 34:932-937.

10.1016/j.ejso.2008.02.004 